\title{
On the general relativistic framework of the Sagnac effect
}

\author{
Elmo Benedetto ${ }^{1, a}$, Fabiano Feleppa ${ }^{2, b}$, Ignazio Licata ${ }^{3,4, c}$, Hooman Moradpour ${ }^{3,4, d}$, Christian Corda ${ }^{3,4, e}$ \\ ${ }^{1}$ Dipartimento di Informatica, Università di Salerno, Via Giovanni Paolo II, 132, 84084 Fisciano, SA, Italy \\ ${ }^{2}$ Department of Physics, University of Trieste, via Valerio 2, 34127 Trieste, Italy \\ ${ }^{3}$ Research Institute for Astronomy and Astrophysics of Maragha (RIAAM), P.O. Box 55134-441, Maragha, Iran \\ ${ }^{4}$ International Institute for Applicable Mathematics and Information Sciences (IIAMIS), B.M. Birla Science Centre, Adarsh Nagar, Hyderabad \\ 500463 , India
}

Received: 21 January 2019 / Accepted: 16 February 2019 / Published online: 4 March 2019

(C) The Author(s) 2019

\begin{abstract}
The Sagnac effect is usually considered as being a relativistic effect produced in an interferometer when the device is rotating. General relativistic explanations are known and already widely explained in many papers. Such general relativistic approaches are founded on Einstein's equivalence principle (EEP), which states the equivalence between the gravitational "force" and the pseudo-force experienced by an observer in a non-inertial frame of reference, included a rotating observer. Typically, the authors consider the so-called Langevin-Landau-Lifschitz metric and the path of light is determined by null geodesics. This approach partially hides the physical meaning of the effect. It seems indeed that the light speed varies by $c \pm \omega r$ in one or the other direction around the disk. In this paper, a slightly different general relativistic approach will be used. The different "gravitational field" acting on the beam splitter and on the two rays of light is analyzed. This different approach permits a better understanding of the physical meaning of the Sagnac effect.
\end{abstract}

\section{Introduction}

It can be useful to recall the context of the discovery of the Sagnac Effect. At the beginning of previous century, physicists were engaged in a very long debate concerning absolute space and its counterpart, the aether, the hypothetical medium of propagation of light. In the well known gedankenexperiment of the rotating bucket filled with water, Newton deduced the existence of an absolute rotation with respect to absolute space. In one of the most important work in the history of sci-

\footnotetext{
a e-mail: elmobenedetto@libero.it

b e-mail: feleppa.fabiano@gmail.com

ce-mail: ignazio.licata3@gmail.com

de-mail: hn.moradpour@gmail.com

e e-mail: cordac.galilei@gmail.com
}

ence (Principia), he expatiated on time, absolute and relative space and motion [1]. Mach criticized Newton's reasoning in his book published in 1893 [2]. From his perspective, one must consider the rotation of water relative to all the matter in the Universe. It is well known that Mach's ideas had a considerable influence on the development of Albert Einstein's general theory of relativity (GTR), especially during the first years of the 20th century. Mach's view led to a misconception about the GTR. A more complete analysis of the debate can be find in [3]. After the formulation of the special theory of relativity and before its generalization to the GTR, also the French physicist Georges Sagnac took part in the debate. In 1899 , he indeed developed a theory of the existence of a motionless mechanical aether [4]. His aim was to explain all optics phenomena within this theoretical framework, with special attention to the Fresnel-Fizeau experiment for the drag of light in a moving medium [5,6]. At the beginning of the 20th century, he conceived a rotating interferometer to test his ideas. Despite countless explanations, in more than a hundred years, there are still different interpretations of Sagnac experiment in the framework of the GTR. But this is not a rare thing in physics. In fact, it is not the only topic that, although it is well known in the scientific literature, still requires insights and explanations $[7,8]$. In order to start, in next Sections, the Sagnac effect in the framework of Classical Mechanics will be briefly analyzed.

\section{The Sagnac experiment within the framework of Classical Mechanics}

One considers two light rays in opposite directions around a static circular loop of radius $r$. Such light rays will arrive at the end point simultaneously. Instead, if the loop is rotating, the ray travelling in the same direction as the rotation of the loop must travel a distance greater than the ray travelling in 
the opposite direction. For this reason, the counter-rotating ray will arrive earlier than the co-rotating ray. The length of the path is $L=2 \pi r$ and, if there is not angular velocity of the loop, the duration of the path is

$\Delta t=\frac{2 \pi r}{c}$.

Instead, in the presence of an angular velocity $\omega \neq 0$, one writes

$c \Delta t_{1}=2 \pi r+r \omega \Delta t_{1}$,

$c \Delta t_{2}=2 \pi r-r \omega \Delta t_{2}$,

from which one obtains

$$
\begin{aligned}
\Delta t_{1} & =\frac{2 \pi r}{c-r \omega}, \\
\Delta t_{2} & =\frac{2 \pi r}{c+r \omega} .
\end{aligned}
$$

Assuming $\omega^{2} r^{2} \ll c^{2}$, the difference in the journey times is

$\Delta t=\Delta t_{1}-\Delta t_{2}=\frac{4 \pi r^{2} \omega}{c^{2}-\omega^{2} r^{2}} \simeq \frac{4 \pi r^{2} \omega}{c^{2}}$.

\section{The Sagnac effect within the framework of the GTR}

The scientific literature on the relativistic Sagnac effect is very wide, see [8-26] for details. In this paragraph, its standard derivation in the framework of the GTR will be considered. Let us recall the standard flat Lorentz-Minkowski metric in cylindrical coordinates

$d s^{2}=c^{2} d t^{2}-d r^{2}-r^{2} d \theta^{2}-d z^{2}$.

If one considers a system rotating at angular velocity $\omega$, one gets the angle transform as $\theta=\theta^{\prime}+\omega t$. Thus, $d \theta=d \theta^{\prime}+$ $\omega d t$. Starting from these considerations, the metric becomes the so called Langevin-Landau-Lifschitz metric [27-29]

$d s^{2}=\left(c^{2}-\omega^{2} r^{2}\right) d t^{2}-d r^{2}-r^{2} d \theta^{\prime 2}-d z^{2}-2 r^{2} \omega d \theta^{\prime} d t$.

Inserting the condition of null geodesics $d s=0$ in Eq. (8), one gets

$$
\left(1-\frac{\omega^{2} r^{2}}{c^{2}}\right) c^{2} d t^{2}-d r^{2}-r^{2} d \theta^{\prime 2}-d z^{2}-2 r^{2} \omega d \theta^{\prime} d t=0 .
$$

Equation (8) describes a stationary metric which is a solution of Einstein field equations in empty space. The EEP permits to interpret it in terms of a gravitational field [27]. Besides, knowing how tensors behave, one has

$R_{i j k l}(t, x, y, z)=0 \Rightarrow R_{i j k l}\left(t, r, \theta^{\prime}, z\right)=0$,

where $R_{i j k l}$ is the Riemann curvature tensor. Following [30], the spatial metric can be written as

$d l^{2}=\left(-g_{\alpha \beta}+\frac{g_{0 \alpha} g_{0 \beta}}{g_{00}}\right) d x^{\alpha} d x^{\beta}$.

Hence, a bit of algebra gives

$d l^{2}=\left(d r^{2}+d z^{2}+\frac{r^{2} d \theta^{2}}{1-\frac{\omega^{2} r^{2}}{c^{2}}}\right)$.

Considering plane motion, one sets $d z=0$ and, finally, one obtains

$d l=\frac{r d \theta^{\prime}}{\sqrt{1-\frac{\omega^{2} r^{2}}{c^{2}}}}$.

Thus, by integrating Eq. (13), the length of the circumference is easily written down as

$l=\int_{0}^{2 \pi} \frac{r d \theta^{\prime}}{\sqrt{1-\frac{\omega^{2} r^{2}}{c^{2}}}}=\frac{2 \pi r}{\sqrt{1-\frac{\omega^{2} r^{2}}{c^{2}}}}$.

Within the platform, the observer on the beam splitter expects both rays to arrive in a time $t=\frac{l}{c}$. At this point, generally one studies the spacetime metric

$d s^{2}=\left(c^{2}\right) d t^{2}-2 r^{2} d \theta^{\prime} \omega d t-r^{2} d \theta^{\prime 2}$,

and the path of the light rays is determined through the condition of null geodesics $d s^{2}=0$. This condition gives

$$
\begin{aligned}
d t & =\frac{r^{2} \omega d \theta^{\prime} \pm \sqrt{r^{4} \omega^{2} d \theta^{\prime 2}+c^{2} r^{2} d \theta^{\prime 2}}}{c^{2}} \\
& =\frac{r^{2} \omega d \theta^{\prime} \pm r^{2} d \theta^{\prime} \sqrt{\omega^{2}+\frac{c^{2}}{r^{2}}}}{c^{2}}
\end{aligned}
$$

which is well approximated by

$d t \approx \frac{r^{2} \omega d \theta^{\prime}\left(\omega \pm \frac{c}{r}\right)}{c^{2}}$.

Then, one gets the solutions

$d t_{1}=\frac{r^{2} \omega+c r}{c^{2}}$

$d t_{2}=\frac{r^{2} \omega-c r}{c^{2}}$. 
By integrating on the periphery of the disk and by observing that $d t_{1}>0$ for $d \theta^{\prime}>0$ and $d t_{2}>0$ for $d \theta^{\prime}<0$, one gets

$t_{1}=\frac{2 \pi r}{c}+\frac{2 \pi r^{2} \omega}{c^{2}}$

$t_{2}=\frac{2 \pi r}{c}-\frac{2 \pi r^{2} \omega}{c^{2}}$.

Then, the time difference is

$t_{1}-t_{2}=\frac{4 \pi r^{2} \omega}{c^{2}}$.

\section{Coordinate velocity of light}

The analogy with radial motion gives simpler calculations. In this case, the metric becomes

$d s^{2}=\left(1-\frac{\omega^{2} r^{2}}{c^{2}}\right) c^{2} d t^{2}-d r^{2}$.

Considering a photon which directed from the center $O$ to a point infinitely near, the condition of null geodesics $d s=0$ permits to obtain that temporal coordinate required for this as

$c d t=\frac{d r}{\sqrt{1-\frac{\omega^{2} r^{2}}{c^{2}}}}$.

The photon on the rim corresponds to

$c t=\int_{0}^{r} \frac{d r}{\sqrt{1-\frac{\omega^{2} r^{2}}{c^{2}}}}$

If $\frac{\omega r}{c} \ll 1$, one gets

$t \simeq \frac{r}{c}+\frac{\omega^{2} r^{3}}{6 c^{3}}+\cdots$

for the coordinate time.

Therefore, if one considers the laboratory clock, the photon's flight lasts longer than $\frac{r}{c}$. In fact, from

$\left(1-\frac{\omega^{2} r^{2}}{c^{2}}\right) c^{2} d t^{2}-d r^{2}=0$

one sees that the coordinate velocity of light decreases with the distance from the center

$\frac{d r}{d t}=c \sqrt{1-\frac{\omega^{2} r^{2}}{c^{2}}}$.
Of course, this is an apparent effect due to time dilation along the path but the local velocity of light is always $c$. Indeed,

$$
\frac{d r}{d \tau}=\frac{d r}{d t} \frac{d t}{d \tau}=c \sqrt{1-\frac{\omega^{2} r^{2}}{c^{2}}} \frac{1}{\sqrt{1-\frac{\omega^{2} r^{2}}{c^{2}}}}=c
$$

\section{Coriolis time delay}

The Coriolis force has a general relativistic explanation. In [32], a general relativistic analysis permits indeed to determine the force on an observer moving with a uniform velocity in a coordinate system which rotates with a constant angular velocity $\omega \neq 0$ as

$\vec{F}=-\frac{m \wedge(\vec{\omega} \wedge \vec{r})+2 m(\vec{\omega} \wedge \vec{v})}{1-\frac{v^{2}}{c^{2}}}$,

where $\vec{v}$ is the velocity of the observer in the rotating system, $\overrightarrow{v^{\prime}}=\vec{v}+(\vec{\omega} \wedge \vec{r})$ is the total velocity of the observer relative to the non-rotating system, and $m$ is the total mass of the observer in the rotating system, see [32] for details. For non-relativistic velocities $\left(v^{\prime} \ll c\right)$ Eq. (28) reduces to [32]

$\vec{F} \simeq-m \wedge(\vec{\omega} \wedge \vec{r})-2 m(\vec{\omega} \wedge \vec{v})$,

where

$\vec{F}_{c}=-m \wedge(\vec{\omega} \wedge \vec{r})$

is the and centrifugal force on the observer and

$\vec{F}_{C}=-2 m \wedge(\vec{\omega} \wedge \vec{v})$

is the Coriolis force. Now, one considers the local Lorentz gauge of the rotating observer [33]. This is the gauge in which the space-time is locally flat and the distance between any two points is given simply by the difference in their coordinates in the sense of Newtonian physics, [33]. In this gauge, "gravitation" manifests itself by exerting "tidal forces" on the masses. Equivalently we can say that there is a "gravitational" potential [33]

$V=\vec{v} \cdot(\vec{\omega} \wedge \vec{r})$,

which generates the the Coriolis "tidal force" of Eq. (31), and that the motion of the test mass is governed by the Newtonian equation

$\ddot{\vec{r}}=-\nabla V$. 
As we are considering a circular motion on the rotating platform, we simply have $V=v \omega r$. Thus, one considers the time dilatation in the weak field approximation by using a well known formula which connects the Newtonian approximation with the linearized GTR [30]

$d \tau=\sqrt{\left(1+\frac{2 V}{c^{2}}\right)} d t \simeq\left(1+\frac{V}{c^{2}}\right) d t=\left(1+\frac{v \omega r}{c^{2}}\right) d t$.

The time delay between the beam splitter and the light rays is

$$
\begin{aligned}
d \tau_{1} & =\left(1+\frac{v \omega r}{c^{2}}\right) d t \\
& =\left(1+\frac{v \omega r}{c^{2}}\right) \frac{r d \theta}{v}=\left(\frac{r}{v}+\frac{\omega r^{2}}{c^{2}}\right) d \theta \\
d \tau_{2} & =\left(1-\frac{v \omega r}{c^{2}}\right) d t \\
& =\left(1-\frac{v \omega r}{c^{2}}\right) \frac{r d \theta}{v}=\left(\frac{r}{v}-\frac{\omega r^{2}}{c^{2}}\right) d \theta .
\end{aligned}
$$

The two Eq. (35) can be integrated as

$$
\begin{aligned}
\tau_{1} & =\int_{0}^{2 \pi}\left(\frac{r}{v}+\frac{\omega r^{2}}{c^{2}}\right) d \theta=\frac{2 \pi r}{v}+\frac{2 \pi r^{2} \omega}{c^{2}} \\
\tau_{2} & =\int_{0}^{2 \pi}\left(\frac{r}{v}-\frac{\omega r^{2}}{c^{2}}\right) d \theta=\frac{2 \pi r}{v}-\frac{2 \pi r^{2} \omega}{c^{2}} .
\end{aligned}
$$

Thus,

$\tau_{1}-\tau_{2}=\frac{4 \pi r^{2} \omega}{c^{2}}$

\section{Conclusions}

In this paper some considerations about the Sagnac experiment have been made. It has been shown that, by considering the rotating metric and by imposing the cancellation of the line element, one has an unexceptionable explanation only from the mathematical point of view. In this way, it seems that the speed of light varies by $c \pm \omega r$ in one or the other direction around the disk. Instead, as it happens for example in Rindler or Schwarzschild metric, the apparent variation of the speed of light is a consequence of time dilation. For this reason, it seems that the physics of the experiment is clearer by using the "gravitational" Coriolis time dilation.

Acknowledgements The Authors thank an unknown Referee for useful comments. This work has been supported financially by the Research Institute for Astronomy and Astrophysics of Maragha (RIAAM), project number 1/6025-63.
Data Availability Statement This manuscript has no associated data or the data will not be deposited. [Author's comment. This is a theoretical work. Thus, there are no raw experimental data to be deposited.]

Open Access This article is distributed under the terms of the Creative Commons Attribution 4.0 International License (http://creativecomm ons.org/licenses/by/4.0/), which permits unrestricted use, distribution, and reproduction in any medium, provided you give appropriate credit to the original author(s) and the source, provide a link to the Creative Commons license, and indicate if changes were made. Funded by $\mathrm{SCOAP}^{3}$.

\section{References}

1. I. Newton, Philosophiae Naturalis Principia Mathematica, Definitiones, Scholium, Imprimatur, S. PEPYS. Reg. Soc. PRAESES, LONDINI, (1686)

2. E. Mach, Die Mechanik in ihrer Entwicklung Historisch-Kritisch Dargerstell (Brockhaus, Leipzig, 1883)

3. E. Benedetto, F. Feleppa, Underlining some mathematical and physical aspects about the concept of motion in general relativity. Afr. Mat. 29, 349 (2018)

4. G. Sagnac, Theorie nouvelle des phenomenes optiques d'entrainement de l'ether par la matiere. Comptes Rendus 129, 818 (1899)

5. G. Sagnac, The demonstration of the luminiferous aether by an interferometer in uniform rotation. Comptes Rendus 157, 708 (1913)

6. H. Fizeau, Sur les hypothèses relatives à l'éther lumineux. Comptes Rendus 33, 349 (1851)

7. E. Benedetto, A. Feoli, Underlining some aspects of the equivalence principle. Eur. J. Phys. 38, 055601 (2017)

8. I. Licata, E. Benedetto, The charge in a lift. A covariance problem. Gravit. Cosmol 24, 173 (2018)

9. F. Tamburini et al., Radiation from charged particles due to explicit symmetry breaking in a gravitational field. Int. J. Geom. Methods Mod. Phys. 15, 07 (2018)

10. A. Ashtekar, A. Magnon, The Sagnac effect in general relativity. J. Math. Phys. 16, 2 (1975)

11. A.A. Logunov, Y.V. Chugreev, Special theory of relativity and the Sagnac effect. Sov. Phys. Uspekhi 31, 9 (1988)

12. G. Rizzi, A. Tartaglia, Speed of light on rotating platforms. Found. Phys. 28, 1663 (1998)

13. G.B. Malykin, Sagnac effect in a rotating frame of reference. Relativistic Zeno paradox. Phys. Uspekhi 45, 907 (2002)

14. G. Rizzi, M.L. Ruggiero, The Sagnac phase shift suggested by the Aharonov-Bohm effect for relativistic matter beams. Gen. Relativ. Gravit. 35, 1745 (2003)

15. G. Rizzi, M.L. Ruggiero, A direct kinematical derivation of the relativistic Sagnac effect for light or matter beams. Gen. Relativ. Gravit. 35, 2129 (2003)

16. G.B. Malykin, The Sagnac effect: Correct and incorrect explanations. Phys. Uspekhi 43, 1229 (2007)

17. G. Rizzi, ML. Ruggiero, (Editors), "Relativity in Rotating Framesin Fundamental Theories of Physics", Series, edited by A. Van derMerwe Kluwer Academic Publishers, Dordrecht (2003)

18. M.L. Ruggiero, A. Tartaglia, A note on the Sagnac effect and current terrestrial experiments. Eur. Phys. J. Plus 129, 126 (2014)

19. G.B. Malykin, V.I. Pozdnyakova, Quadratic Sagnac effect - the influence of the gravitational potential of the Coriolis force on the phase difference between the arms of a rotating Michelson interferometer (an explanation of D C Miller's experimental results, 1921-1926). Phys. Uspekhi 58, 4 (2015) 
20. A. Tartaglia, M.L. Ruggiero, The Sagnac effect and pure geometry. Am. J. Phys. 83, 427 (2015)

21. E. Schwartz, Sagnac effect in an off-center rotating ring frame of reference. Eur. J. Phys. 38, 1 (2016)

22. E. Schwartz, White light Sagnac interferometer - a common (path) tale of light. Eur. J. Phys. 38, 6 (2017)

23. G. Pascoli, The Sagnac effect and its interpretation by Paul Langevin. C. R. Phy. 18, 9-10 (2017)

24. M. Gogberashvili, Coriolis force and Sagnac effect. Found. Phys. Lett. 15, 5 (2002)

25. E. Minguzzi, Simultaneity and generalized connections in general relativity. Class. Quantum Gravity 20, 2443 (2003)

26. A. Rincon, J.R. Villanueva, The Sagnac effect on a scale-dependent rotating BTZ black hole background. (2019). arXiv:1902.03704

27. C. Corda, New proof of general relativity through the correct physical interpretation of the Mossbauer rotor experiment. Int. J. Mod. Phys. D 27, 1847016 (2018)
28. N. Ashby, Relativity in the global positioning system. Living Rev. Relativ. 6, 1 (2003)

29. G. Iovane, E. Benedetto, Coordinate velocity and desynchronization of clocks. Ann. Phys. 403, 106 (2019)

30. L. Landau, E. Lifsits, -Classical Theory of Fields (3rd ed.). London: Pergamon. ISBN 0-08-016019-0. Vol. 2 of the Course of Theoretical Physics (1971)

31. LD. Landau, EM. Lifshitz, Mechanics: Vol. 1 of: Course of Theoretical Physics, Butterworth-Heinemann (1976)

32. C.T. Ridgely, Forces in general relativity. Eur. J. Phys. 31, 4 (2010)

33. C.W. Misner, K.S. Thorne, J.A. Wheeler, Gravitation (W H Feeman and Company, New York, 1973) 